\title{
COMPARISON OF A FLUID AND A SOLID APPROACH FOR THE NUMERICAL SIMULATION OF FRICTION STIR WELDING WITH A NON-CYLINDRICAL PIN
}

\author{
P. Bussetta*, N. Dialami ${ }^{\dagger}$, R. Boman*, M. Chiumenti ${ }^{\dagger}$, \\ C. Agelet de Saracibar ${ }^{\dagger}$, M. Cervera ${ }^{\dagger}$ and J-P. Ponthot* \\ * University of Liege, Department of Aerospace \& Mechanical Engineering, \\ Non Linear Computational Mechanics, Building B52/3, \\ Chemin des Chevreuils, 1; B-4000 Liege, Belgium \\ e-mail: P.Bussetta@ulg.ac.be; R.Boman@ulg.ac.be; JP.Ponthot@ulg.ac.be \\ $\dagger$ International Centre for Numerical Methods in Engineering (CIMNE) \\ Universidad Politécnica de Cataluña \\ Campus Norte UPC, 08034 Barcelona, Spain \\ e-mail: narges@cimne.upc.edu; michele@cimne.upc.edu; agelet@cimne.upc.edu; \\ miguel.cervera@upc.edu
}

Key words: Friction Stir Welding (FSW), Finite Element Method, Remeshing, Arbitrary Lagrangian Eulerian (ALE) formalism

\begin{abstract}
Friction Stir Welding (FSW) process is a solid-state joining process during which materials to be joined are not melted. As a consequence, the heat-affected zone is smaller and the quality of the weld is better with respect to more classical welding processes. Because of extremely high strains in the neighbourhood of the tool, classical numerical simulation techniques have to be extended in order to track the correct material deformations. The Arbitrary Lagrangian Eulerian (ALE) formulation is used to preserve a good mesh quality throughout the computation. With this formulation the mesh displacement is independent from the material displacement. Moreover, some advanced numerical techniques such as remeshing or a special computation of transition interface is needed to take into account non-cylindrical tools. During the FSW process, the behaviour of the material in the neighbourhood of the tool is at the interface between solid mechanics and fluid mechanics. Consequently, a numerical model of the FSW process based on a solid formulation is compared to another one based on a fluid formulation. It is shown that these two formulations essentially deliver the same results in terms of pressures and temperatures.
\end{abstract}




\section{Introduction}

Friction Stir Welding (FSW) is a relatively recent welding process, which was developed at the Welding Institute (UK) in 1991. FSW is a solid-state joining process. It means that during welding the materials to be joined are not melted. The joining is constituted by mechanical intermixing of the two materials. A rotating non-consumable tool is inserted between the two work-pieces and displaced along the welding direction (see figure 1). The tool is composed of two parts: a pin and a shoulder. The pin is introduced into the welded joint to mix deeply the two materials together. The aim of the shoulder is to contain the material around the pin. The part of the welding joint where the velocity of the tool and the advance velocity add up is named the advancing side. The other part, where the two velocities are in opposite directions, is called the retreating side. The friction between the rotating tool and the work-pieces as well as the plastic deformation in the neighbourhood of the tool increase the temperature in the welded zone. But, during the process, the temperature is always smaller than the melting temperature of the materials. So, the heat-affected zone is smaller and the quality of the welding is higher than in more classical welding processes.

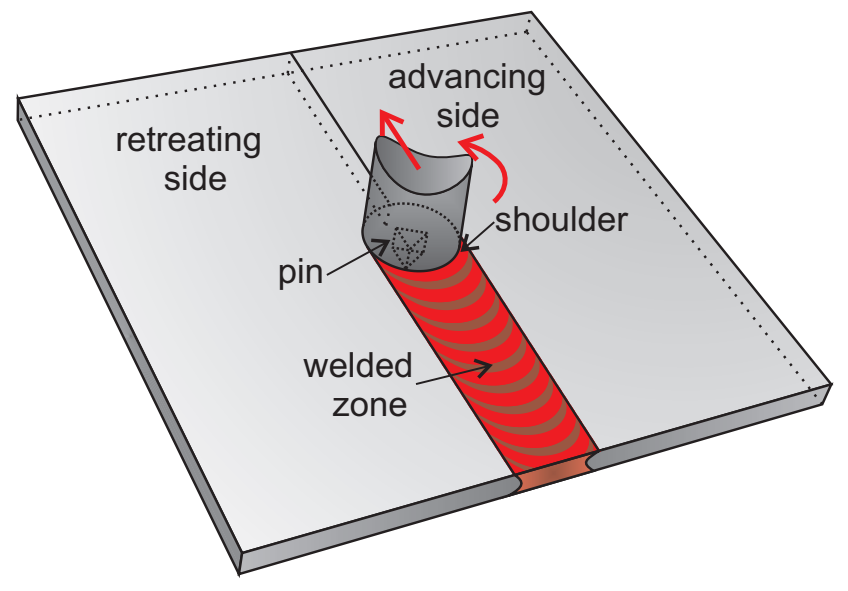

(a) General view

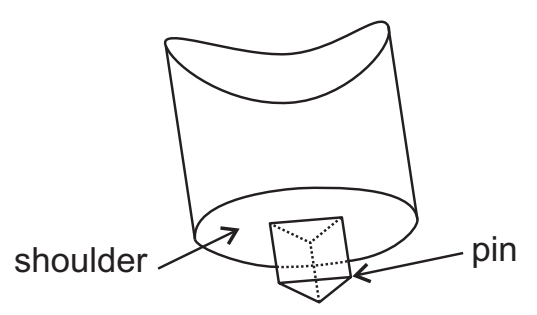

(b) View of the tool

Figure 1: Scheme of the FSW process.

In spite of the important number of applications of FSW, the phenomena happening during welding are still not very well understood. Therefore, the investigations on this process and especially regarding numerical simulations are still very active $[1,2,3]$. The mechanical intermixing submits the material in the neighbourhood of the tool to extremely high strains. Consequently, classical numerical simulation techniques have to be extended in order to track the correct material deformations. One of these possible extended techniques is the Arbitrary Lagrangian Eulerian (ALE) formulation [4, 5, 6]. 
This formulation is used to control the mesh motion regardless of the real material displacements. The ALE formulation is also used to maintain a good mesh quality during the computation. For that sake, in the general case of a non-cylindrical pin, the ALE formulation has to be enhanced and advanced numerical techniques such as a remeshing operator step is needed.

This paper presents and compares two different numerical models of the FSW process. The first model is based on a solid approach written in terms of nodal positions and nodal temperatures. The second model of FSW process is based on a fluid approach written in terms of the velocity, the pressure and the temperature fields. Both models use advanced numerical techniques such as remeshing and the ALE formulation.

\section{2D Numerical modelling of FSW process}

The FSW process is modelled in two dimensions under the plane strain hypothesis. To model this welding process, the displacement of the tool is split into an advancing movement (actually assigned to the work-pieces but, in the opposite direction) and a rotation (imposed to the tool).

In other words, the centre of the pin is fixed and a constant velocity is imposed to the plates (see figure 2(a)). The tool is described by a classical Lagrangian mesh. Then, in relation with the distance to the centre of the tool, three zones of the plates are identified. In the closest zone around the pin, the material is submitted to extremely high strains. This region is called the thermo-mechanically affected zone (TMAZ). Due to high deformations, the use of a Lagrangian formalism would lead very quickly to mesh entanglement. Thus, in this region, the ALE formulation is employed. On top of this, the ALE formulation allows the model to take into account non-circular pin shapes. In this zone, the mesh has the same rotational speed as the pin (red region in figure 2(b)). In the furthest zone from the tool, the grey zone in figure 2(b), the Eulerian formulation is used. Thus in this region, the mesh is fixed. The ring connecting region 1 and region 3 is a transition zone (white region numbered 2 in figure 2(b)). In such a model, the quality of the mesh does not change during the simulation except in the transition zone. So, to overcome this problem, two different numerical techniques are proposed (see section 2.2.2).

\subsection{Thermomechanical formulation}

The numerical models presented here are based on the finite element method. The thermomechanical equations are split in a mechanical part and a thermal part. First, the mechanical equations are solved for the temperature field computed at the latest increment. Then, the thermal equations are solved on the frozen resulting geometrical configuration. In this paper, two numerical formulations are compared. The first one is based on a solid mechanics approach. It is written in terms of nodal positions and temperatures. The second one is based on a fluid mechanics approach. The equilibrium 


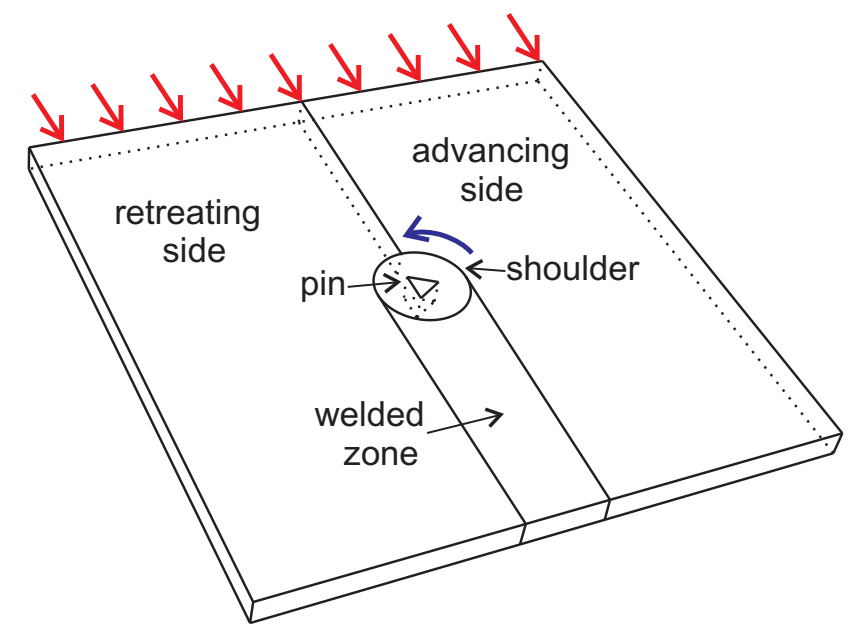

(a) Scheme of the FSW model. A rotation is imposed to the pin (blue arrow) while the advancing velocity of the pin is replaced by a velocity imposed to the plates in the opposite direction (red arrows).

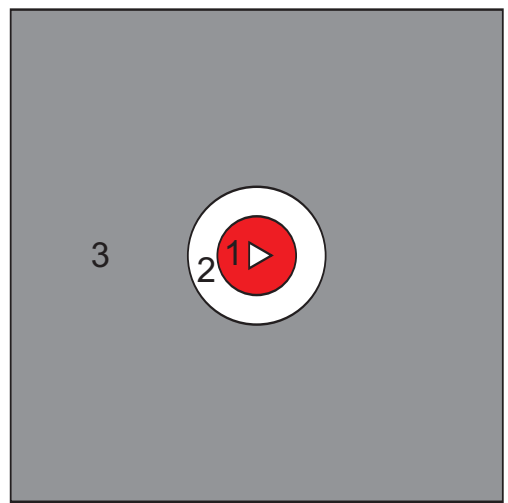

(b) The different zones of the model: ALE formulation is used on the red region (1), the transition zone corresponds to the white region (2), and the Eulerian formulation is applied on the grey region (3)

Figure 2: Description of the FSW model

is written as a function of nodal velocities, pressures and temperatures.

Solid approach In the solid approach, the finite element used are linear quadrilaterals. The position and temperature fields are computed at each node of the elements. The stresses and the internal variables are computed at each quadrature point of the element (4 Gauss points). To overcome the locking phenomenon, the pressure is considered constant over the element and computed only at a central quadrature point.

Fluid approach The fluid approach is based on a stabilized mixed linear velocitypressure finite element formulation. A triangular mesh is used for the domain discretization. The velocity, the pressure and temperature fields are computed at each node of the element. The deviatoric stresses and the other internal variables are computed at each quadrature point of the element. This approach is exposed in more details in $[1,2,7,8]$.

\subsection{Numerical simulation strategy}

\subsubsection{Arbitrary Lagrangian Eulerian formulation}

In region 1 and region 3 in figure 2(b) the Arbitrary Lagrangian Eulerian formulation is used. Indeed, the Eulerian formulation (used in the region 3) is a particular case of 
the ALE formulation. With the ALE formalism, the mesh displacement can be decoupled from the material displacement $[1,2,4,5,6]$. The ALE formulations used in the two approaches are different.

Solid approach The ALE formulation used in the solid approach is described in more details in $[4,5,6]$. To simplify the solution procedure and remain competitive against Lagrangian models, the system of ALE equations is solved using an operator-split procedure. First, for each time step, the classical Lagrangian formalism is used. During this Lagrangian step the mesh sticks to the material until an equilibrated Lagrangian configuration is iteratively obtained. The second step, also called the Eulerian step, is divided into two substeps: first the nodes of the mesh are relocated to a more suitable position, thus defining a new mesh. In the case of region 1 and region 3 the position of the relocated nodes is known because the mesh velocity of these regions is imposed. Then, the unknowns and the internal variables are transferred from the old mesh to the new one [6].

Fluid approach The ALE formulation used in the fluid model is not based on a operator-split like in the formulation presented for the solid approach. In this fully coupled formulation [1, 2], the equilibrium state is computed at each time increment without remeshing and remapping steps. The system of equations solved includes the convective terms due to the velocity of the mesh relative to the material. In the TMAZ, region 1 in figure 2(b), the velocity of the mesh is imposed and the velocity and the pressure of the material are directly computed. In the case of the Eulerian formulation (region 3 in figure $2(\mathrm{~b})$ ), the mesh does not move during the computation.

\subsubsection{The transition zone}

Solid approach In the solid approach, the transition zone is a ring with a finite thickness (region 2 in figure 2(b)). In this region, the evolution of the rotational speed of the mesh, which differs from the material velocity, is linearly interpolated between the ALE region and the Eulerian zone. As the mesh distortion grows with time, a remeshing operation is periodically required. For one full rotation of the pin, the remeshing process is applied 30 times. The time interval between two successive remeshings is thus constant.

The remeshing operation can be divided into two steps. First, a better-suited mesh, called the new mesh, is created. In the case of the transition zone, the relatively simple geometry of this region allows an easy generation of the new quadrangular mesh.

Then, to carry on the computation over this mesh, the state variables from the mesh before remeshing, called the old mesh, has to be transferred to the new one. Each field used to define the equilibrium state is transferred independently from the other ones. The data transfer method used in this paper is called the Finite Volume Transfer Method (FVTM) with linear reconstruction of the fields. In $[9,10]$, this transfer method is presented in 
more details and the comparison with some of the remapping algorithms most commonly used in the literature brings to light the advantages of this method.

Fluid approach In the fluid model the transition zone (region 2 in figure 2(b)) is limited to a circle (zero thickness). Each node of the mesh on this circle is duplicated. One node is linked to the ALE region (numbered 1) and the other one to the Eulerian region (numbered 3). The coupling between both regions is performed using a specific node-to-node link approach. At every mesh movement step, for a given node of the ALE region, the corresponding node of the Eulerian one is found and a link between the two nodes is created. Afterwards, the boundary conditions and the properties of the plate nodes are copied to the corresponding TMAZ nodes within the link. The time step can be conveniently chosen such that the two interface meshes (ALE and Eulerian) are always compatible. In this case the ALE mesh would slide precisely from one Eulerian interface node to the next one at each time step.

\subsubsection{Thermomechanical constitutive model}

In both models, the constitutive model of the tool is thermo-rigid. So, no mechanical fields are computed over this material. However, from the point of view of the thermal equations, the tool has a classical thermal behaviour as far as heat conduction is concerned. In addition, the material behaviour of the plates is modelled as thermo-visco-plastic using a Norton-Hoff constitutive model:

$$
\boldsymbol{S}=2 \mu \boldsymbol{D}\left(\sqrt{3} \sqrt{\frac{2}{3} \boldsymbol{D}: \boldsymbol{D}}\right)^{m-1}
$$

where $m$ and $\mu$ are the rate sensitivity and viscosity parameters respectively. Both are temperature dependent. $\boldsymbol{S}$ is the deviatoric part of the stress tensor and $\boldsymbol{D}$ is the deviatoric part of the strain-rate tensor.

Solid approach In the solid model, the value of the variation of the pressure $(d p)$ is computed thanks to the variation of the volume $(d V)$ and the bulk modulus $(K)$ : $d p=K d V$. In addition, with the solid approach, it is possible to replace the Norton-Hoff constitutive model with a thermo-elasto-visco-plastic one, see e.g. [11]. With this kind of constitutive model, it is possible to compute the residual stresses.

Fluid approach In the fluid model, the material is assumed to be incompressible and this constraint is incorporated into the equations to be solved. 


\section{Comparison of numerical results based on the two approaches}

In this example, the section of the pin is an equilateral triangle (figures 3 and 4(a)). The mesh used with the solid model is presented at the beginning of the computation in figure 3. The dimensions of the tool are:

- radius of the circumscribed circle to the pin: $3 \mathrm{~mm}$.

The width of the two plates is $50 \mathrm{~mm}$ and the simulated length is $100 \mathrm{~mm}$. The simulated region of the plates is a square with a side of $100 \mathrm{~mm}$. The centre of the pin is on the centre of this square (see figures 3 and $4(\mathrm{a})$ ).

The most important parameters of the considered FSW process are:

- rotation speed: $40 R P M$

- welding speed: $400 \mathrm{~mm} \mathrm{~min}^{-1}$.

The thermo-mechanical properties of the plates are the following:

- density: $2700 \mathrm{~kg} \mathrm{~m}-3$

- bulk modulus: 69 GPa (used only with the solid approach)

- thermo-mechanical Norton-Hoff law (presented in the part 2.2.3) with $\mu=100 M P a, m=0.12$,

- heat conductivity: $120 \mathrm{~W} \mathrm{~m}^{-1} \mathrm{~K}^{-1}$

- thermal expansion coefficient: $1 \times 10^{-6} K^{-1}$

- heat capacity: $875 \mathrm{~J} \mathrm{~kg}^{-1} \mathrm{~K}^{-1}$

The thermo-mechanical properties of the tool are the following:

- density: $7800 \mathrm{~kg} \mathrm{~m}$

- heat conductivity: $43 \mathrm{~W} \mathrm{~m}^{-1} \mathrm{~K}^{-1}$;

- heat capacity: $460 \mathrm{~J} \mathrm{~kg}^{-1} \mathrm{~K}^{-1}$.

The total time of the simulation is 15 seconds which corresponds to 10 revolutions for the pin.

Figures 4(b), 4(c) and 4(d) show the evolution of the pressure computed by the two models at three reference points defined in figure 4(a). Points 1 and 2 move with the mesh, because these points have the same rotational speed as the pin. On the other hand, 

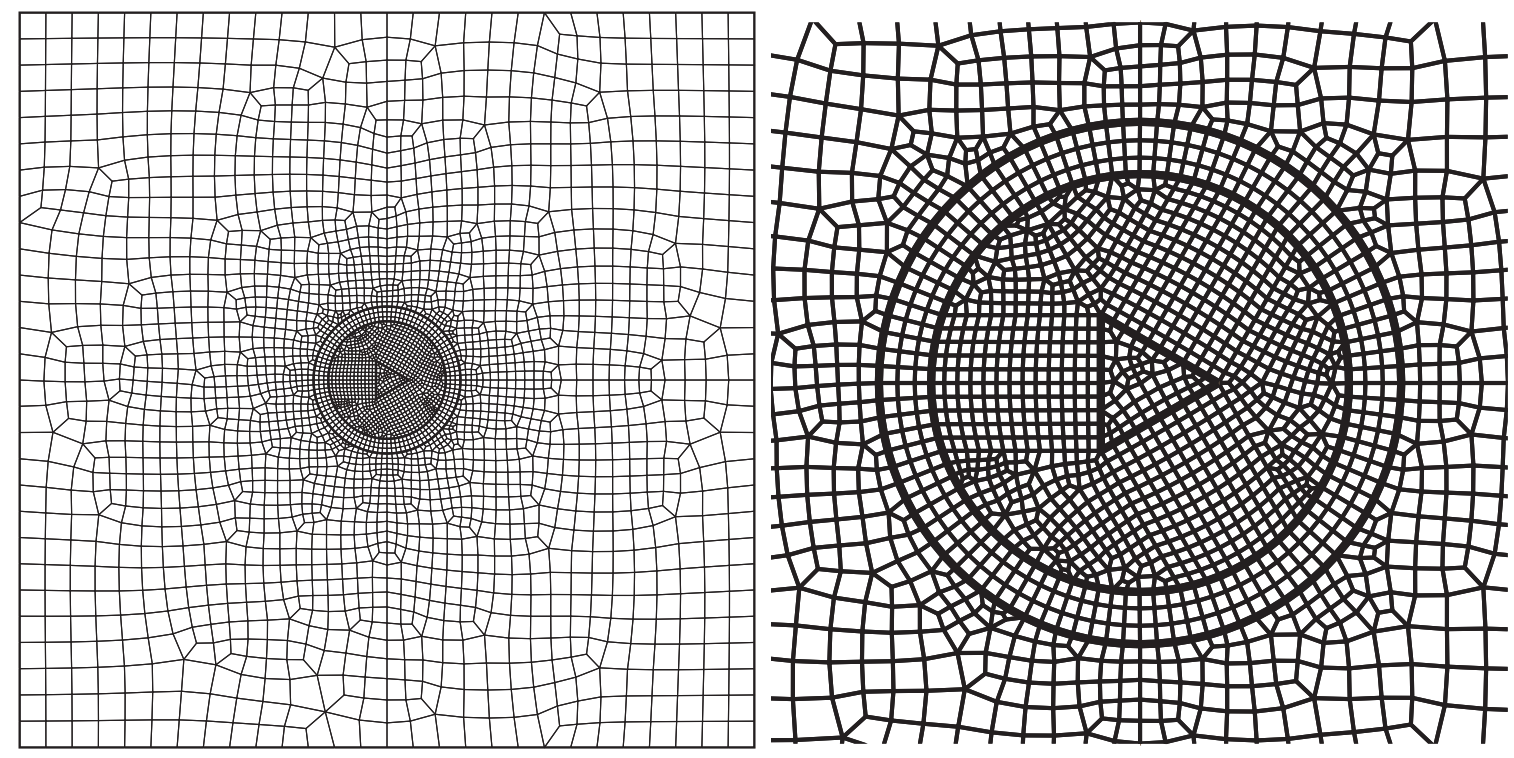

Figure 3: Initial quadrangular mesh (composed of 3237 elements) used in the solid model (global view and zoom)

point 3 is fixed in space. Figure 5 presents the evolution of the temperature computed with the two models at the three reference points. After a transient phase which depends on the numerical strategy adopted for each approach, the results of both models are very similar (see figures 4 and 5). The difference of frequency between the pressure at point 3 and the pressure and the temperature at points 1 and 2 is explained by the fact that point 3 is fixed in space while points 1 and 2 have the same rotational velocity as the pin. On the one hand, the pressure at point 3 is affected by the three corners of the pin. On the other hand, the frequency of the pressure and the temperature at points 1 and 2 are controlled by the rotation speed of the pin. Consequently, the frequency of the pressure at point 3 is three times higher than the frequency of the pressure or the temperature at points 1 or 2 . Figure 6 presents the temperature field at the end of the simulation with the solid model.

\section{Conclusion and future works}

The phenomena happening during the Friction Stir Welding (FSW) process are at the interface between solid mechanics and fluid mechanics. In this paper, two different methods are presented to simulate the FSW process numerically. One model is based on a solid approach which computes the position and the temperature fields and another one is based on a fluid approach written in terms of velocity, pressure and temperature fields. Both models use advanced numerical techniques such as the Arbitrary Lagrangian Eulerian formalism or remeshing operations or an advanced stabilization algorithm. These advanced numerical techniques allow the simulation of the FSW process with non-circular tool 


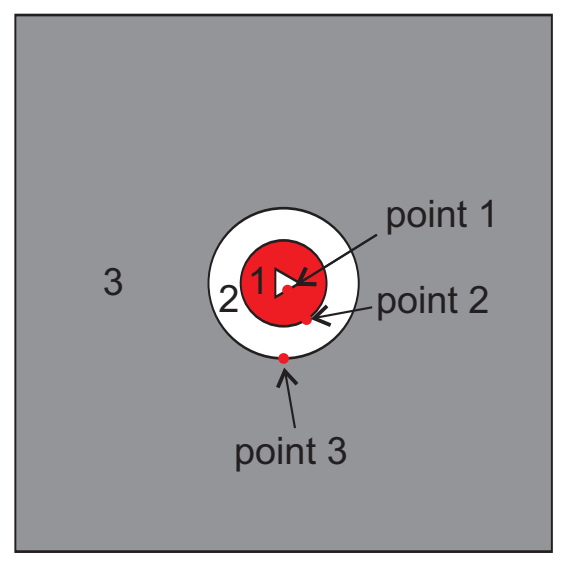

(a) Mesh regions of the simulated FSW model with the initial position of three selected points

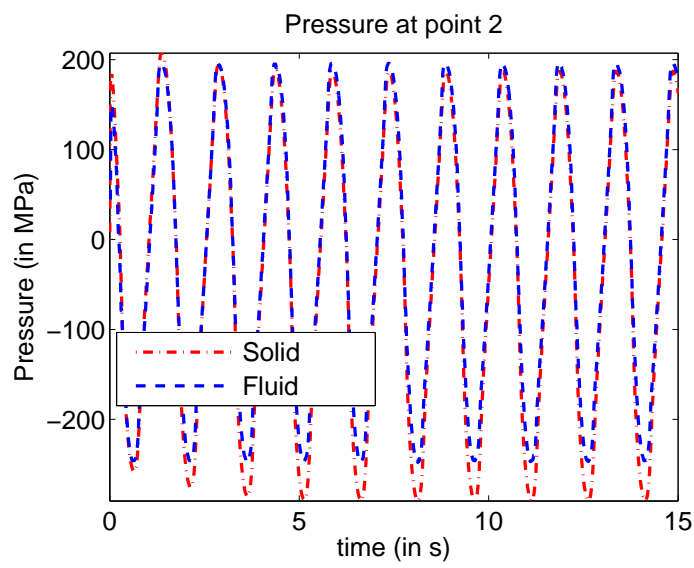

(c) Pressure at point 2 (rotating with the pin)

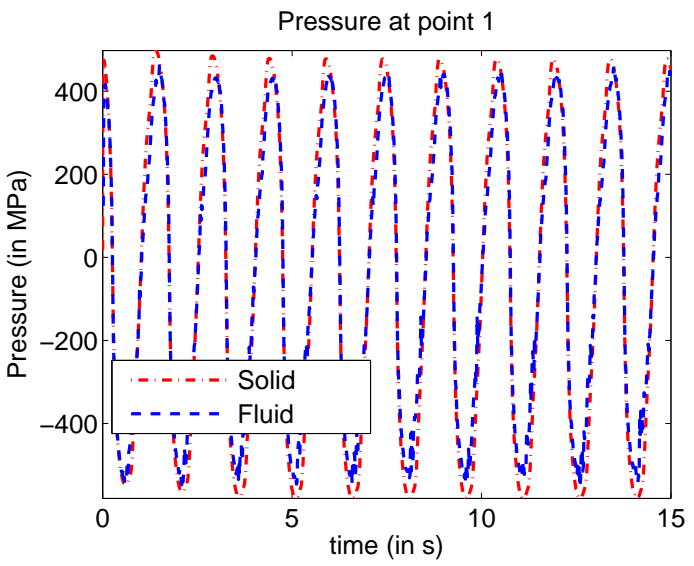

(b) Pressure at point 1 (rotating with the pin)

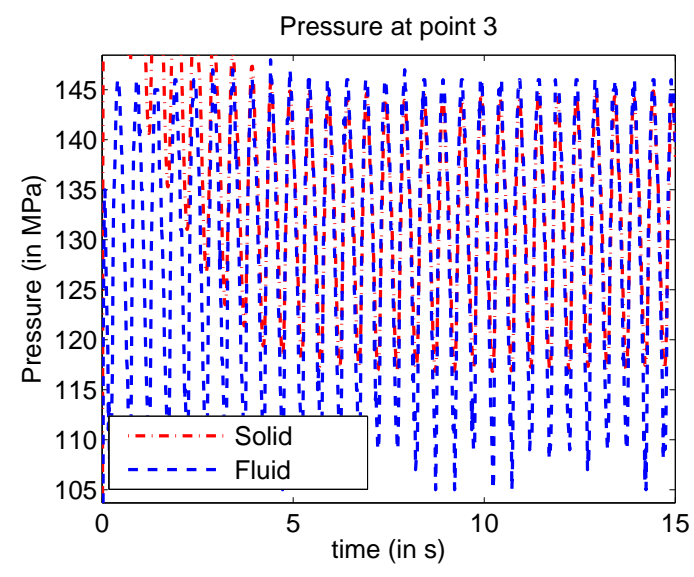

(d) Pressure at point 3 (fixed in space)

Figure 4: Evolution of the pressure computed by the two models at the three reference points

shapes. The presented example (with a triangular pin) shows that the two formulations essentially deliver the same results. More investigations are still needed to understand the small differences between the two models. While the fluid model is more efficient from a computational point of view, the model based on the solid approach has the advantage that it can be used to compute the residual stresses (the thermo-visco-plastic constitutive model can be replaced with a thermo-elasto-visco-plastic one).

\section{Acknowledgements}

The Belgian authors wish to acknowledge the Walloon Region for its financial support to the STIRHETAL project (WINNOMAT program, convention number 0716690) in the 


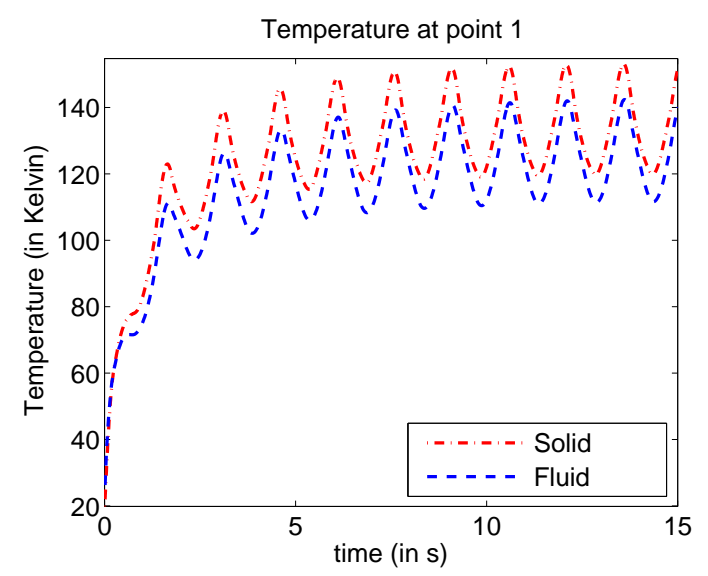

(a) Temperature at point 1 (rotating with the pin)

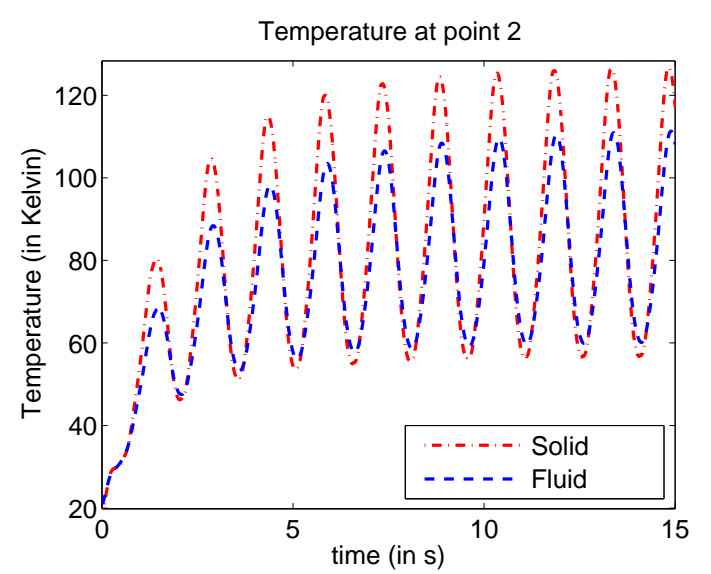

(b) Temperature at point 2 (rotating with the pin)

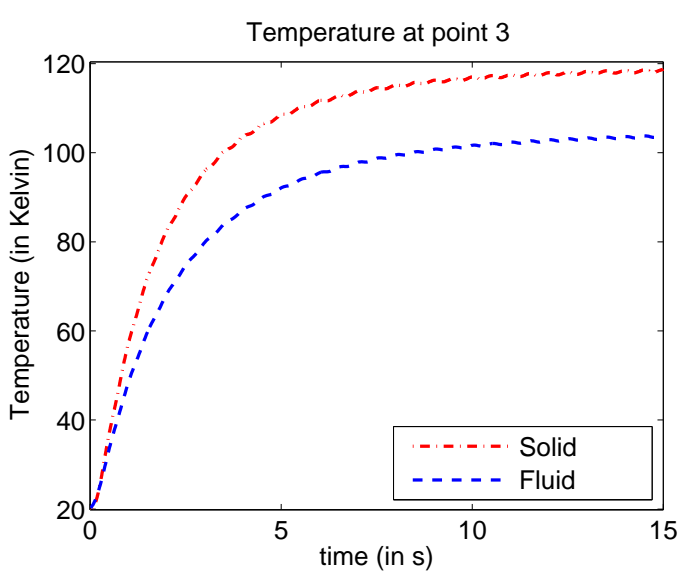

(c) Temperature at point 3 (fixed in space)

Figure 5: Evolution of the temperature computed by the two models at the three reference points

context of which this work was performed.

\section{References}

[1] N. Dialami, M. Chiumenti, M. Cervera, and C. Agelet de Saracibar. An apropos kinematic framework for the numerical modelling of friction stir welding. Computers and Structures, 117:48-57, 2013. doi: 10.1016/j.compstruc.2012.12.006.

[2] M. Chiumenti, M. Cervera, C. Agelet de Saracibar, and N. Dialami. Numerical modeling of friction stir welding processes. Computer Methods in Applied Mechanics and Engineering, 254:353-369, 2013. doi: 10.1016/j.cma.2012.09.013.

[3] M. Assidi, L. Fourment, S. Guerdoux, and T. Nelson. Friction model for friction stir welding process simulation: Calibrations from welding experiments. In- 

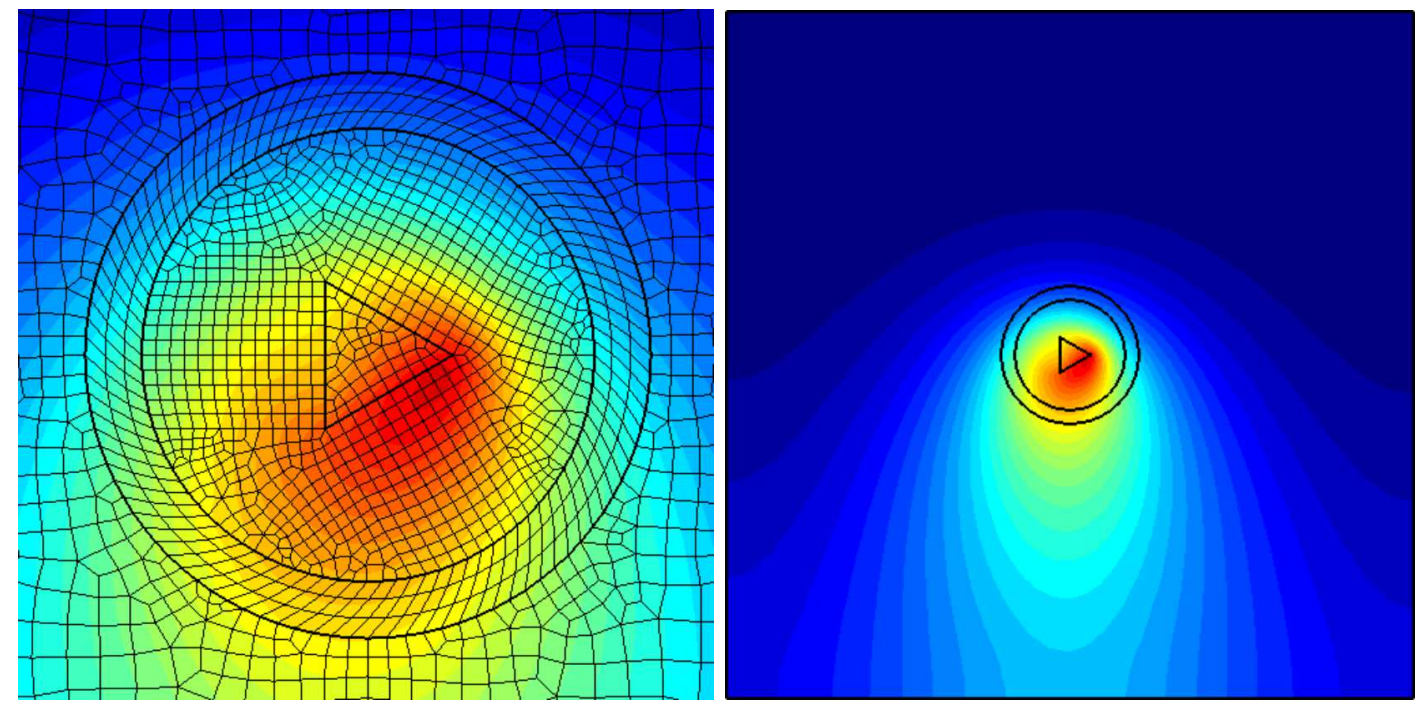

Temperature

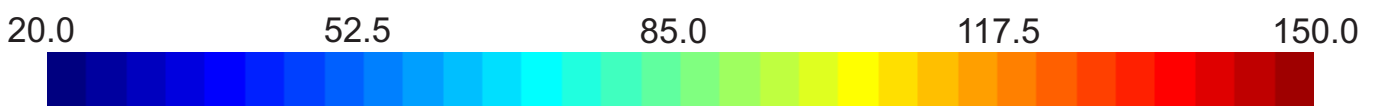

Figure 6: Temperature field (in ${ }^{\circ} \mathrm{C}$ ) at the end of the computation (time : $15 \mathrm{~s}$ ) obtained with the solid model

ternational Journal of Machine Tools and Manufacture, 50(2):143-155, 2010. doi: 10.1016/j.ijmachtools.2009.11.008.

[4] J. Donea, A. Huerta, J.-P. Ponthot, and A. Rodríguez-Ferran. Encyclopedia of Computational Mechanics, chapter Arbitrary Lagrangian-Eulerian Methods. John Wiley \& Sons, Ltd, 2004. doi: 10.1002/0470091355.ecm009.

[5] R. Boman and J.-P. Ponthot. Efficient ALE mesh management for 3D quasi-eulerian problems. International Journal for Numerical Methods in Engineering, 92:857 - 890, 2012. doi: $10.1002 /$ nme.4361.

[6] R. Boman and J.-P. Ponthot. Enhanced ALE data transfer strategy for explicit and implicit thermomechanical simulations of high-speed processes. International Journal of Impact Engineering, 53:62-73, 2013. doi: 10.1016/j.ijimpeng.2012.08.007.

[7] C. Agelet de Saracibar, M. Chiumenti, Q. Valverde, and M. Cervera. On the orthogonal subgrid scale pressure stabilization of finite deformation j2 plasticity. Computer Methods in Applied Mechanics and Engineering, 195:1224-51, 2006. doi: 10.1016/j.cma.2005.04.007.

[8] M. Chiumenti, Q. Valverde, C. Agelet de Saracibar, and M. Cervera. A stabilized 
formulation for incompressible plasticity using linear triangles and tetrahedra. International Journal of Plasticity, 20:1487-1504, 2004. doi: 10.1016/j.ijplas.2003.11.009.

[9] P. Bussetta, R. Boman, and J.-P. Ponthot. Novel three-dimensional data transfer operators using auxiliary finite volume mesh or mortar elements and based over numerical integration. in preparation.

[10] P. Bussetta and J.-P. Ponthot. A finite volume based data transfer method for remeshing. application to metal forming problems. In Proceedings of the 18th International Symposium on Plasticity and Its Current Applications, San Juan, Puerto Rico, January 2012. URL http://hdl .handle.net/2268/100394.

[11] J.P. Ponthot. Unified stress update algorithms for the numerical simulation of large deformation elasto-plastic and elasto-viscoplastic processes. International Journal of Plasticity, 18:91-126, 2002. doi: 10.1016/S0749-6419(00)00097-8. 